\title{
CONGRUENCES FOR $m$-ARY PARTITIONS
}

\section{GUNNAR DIRDAL}

1.

Let $s_{m, q}(n)$ denote the number of partitions of a natural number $n$ into non-decreasing powers of a given natural number $m(m>1)$ of which $m^{q}$ is the maximum.

We put $s_{m, q}(n)=0$ if $n$ is not a non-negative integer and $c_{k}=1$ or $2^{\min (k, q)-1}$ according as $m$ is odd or even. $k$ is a positive integer and $[a]$ denotes the integral part of $a$.

The object of this paper is to prove the following two theorems;

TheORem 1. If there exist $k$ integers $j, 0 \leqq j<q$, such that

then

$$
\left[n / m^{j}\right] \equiv-1 \quad(\bmod m)
$$

$$
s_{m, q}(m n) \equiv 0 \quad\left(\bmod m^{k} / c_{k}\right)
$$

Theorem 2. Let $q>0$. Then

$$
s_{m, q}\left(m^{k+1} n-m\right) \equiv 0 \quad\left(\bmod m^{k} / c_{k}\right) .
$$

The proof of Theorem 1 is based on Theorem 1 in Dirdal [3].

Let $r_{1}, \ldots, r_{l}$ denote the digits in the representation of $n>0$ in the base $m$,

$$
n=\sum_{j=1}^{l} r_{j} m^{j-1} ; \quad 0 \leqq r_{j}<m,\left[n / m^{j-1}\right] \equiv r_{j}(\bmod m) .
$$

Then the conditions in Theorem 1 are satisfied if and only if $k$ of the $q$ first of these digits equal $m-1$.

In particular, if we for some $i \geqq 1$ put

then

$$
r_{j}=m-1 \text { for } j=i, i+1, \ldots, i+k-1 ; i+k-1 \leqq q,
$$

and

$$
n=m^{k+i-1}\left(\left[n / m^{k+i-1}\right]+1\right)-m^{i-1}+t_{i} ; \quad 0 \leqq t_{i}<m^{i-1},
$$

$$
s_{m, q}(m n) \equiv 0 \quad\left(\bmod m^{k} / c_{k}\right)
$$

Received February 5, 1975. 


\section{Hence}

$$
s_{m, q}\left(m^{k+i} n-m^{i}+m t_{i}\right) \equiv 0 \quad\left(\bmod m^{k} / c_{k}\right),
$$

for all $n, i \geqq 1,0 \leqq t_{i}<m^{i-1}$ and $k+i-1 \leqq q$.

If $q=\infty$ Theorem 2 is actually a corollary of Theorem 1 and can be written

$$
s_{m}\left(m^{k+1} n\right)-s_{m}\left(m^{k} n\right) \equiv 0 \quad\left(\bmod m^{k} / c_{k}\right),
$$

when using (2.1) and putting $s_{m, q}=s_{m}$.

This result for unrestricted partions $(q=\infty)$ was first proved for $m=p$, $p$ an odd prime, by Rödseth [5]. Gupta [4] proved it later for $m>2$. Andrews [1] has also proved a result conserning unrestricted partitions, but his result is slightly weaker than the above mentioned. However, it was Churchhouse [2], discussing computer evidence, who first discovered congruence properties for unrestricted partitions.

2.

Put $s_{m, q}(0)=1$ and

$$
G_{m, q}(x)=\sum_{n=0}^{\infty} s_{m, q}(n) x^{n} \quad(|x|<1) .
$$

The generating function of $s_{m, q}(n)$ is

$$
G_{m, q}(x)=\prod_{l=0}^{q}\left(1-x^{m^{l}}\right)^{-1},
$$

and from this it is easily seen that

Hence

$$
G_{m, q}(x)=(1-x)^{-1} G_{m, q-1}\left(x^{m}\right) .
$$

$$
s_{m, q}(n)-s_{m, q}(n-1)=s_{m, q-1}(n / m),
$$

and from (2.1) we deduce

$$
s_{m, q}(m n)=\sum_{l=0}^{n} s_{m, q-1}(l) .
$$

Noticing that $s_{m, 0}(n)=1,(2.2)$ gives $s_{m, 1}(m n)=n+1$, which proves Theorem 1 and 2 for $q=1$. Suppose therefore that $q>1$ in the rest of this paper.

Define the integers $r_{j}=r_{j}(n)$ and $n_{j}=n_{j}(n)$ recursively by

$$
n_{j}=m n_{j+1}+r_{j+1}, \quad n_{0}=n, \quad 0 \leqq r_{j+1}<m .
$$

Hence $\left[n / m^{j}\right]=n_{j}$ and since

$$
\left[\left[n / m^{i}\right] / m^{j-1}\right]=\left[n / m^{i+j-1}\right],
$$

we have $r_{j}\left(n_{i}\right)=r_{j+i}(n)$. 
Dirdal [3] has proved that there exist integers $a_{n, k}(i) ; k \leqq q$; depending on $m$, such that

$$
s_{m, q}(m n) \equiv \sum_{i=1}^{k} a_{n, k}(i) s_{m, q-i}\left(\left[n / m^{i}\right] m\right) \quad\left(\bmod m^{k}\right),
$$

where

$$
\begin{gathered}
a_{n, k}(i) \equiv 0\left(\bmod 2^{\mu(i)-1} m^{i-1}\right) ; \quad \mu(i) \equiv m^{i-1}(\bmod 2), \mu(i)=0,1 \\
\left\{\begin{array}{c}
a_{n, k}(1)=r_{1}+1+\sum_{t=0}^{m-1} a_{t, k-1}(1) \\
a_{n, k}(i)=\sum_{t=0}^{m^{i}-1} a_{t, k-1}(i)+m \sum_{t=0}^{r_{2}+r_{3} m+\ldots+r_{i} m^{i-2}-1} a_{t, k-1}(i-1)- \\
-\left(r_{i}+1\right) \sum_{t=0}^{m^{i-1}-1} a_{t, k-1}(i-1) \quad 2 \leqq i \leqq k
\end{array}\right.
\end{gathered}
$$

and

$$
a_{n, k}(i)=a_{n^{\prime}, k}(i) \quad \text { if } i>1 \text { and }[n / m]=\left[n^{\prime} / m\right] \text {. }
$$

We put $a_{n, k}(i)=0$ if $i>k$.

Now we have the

Lemma. Let $1 \leqq i \leqq k$. If $r_{i}(n)=m-1$ then

$$
a_{n, k}(i) \equiv 0 \quad\left(\bmod 2^{\mu(k)-1} m^{i}\right) .
$$

Proof. For the definition of $\mu(k)$ see (2.4). We use induction on $k$. By (2.5) we get

$$
\sum_{n=0}^{m-1} a_{n, k}(1)=\frac{m(m+1)}{2} \frac{m^{k}-1}{m-1}
$$

Hence

$$
a_{n, k}(1)= \begin{cases}r_{1}+1 & \text { if } k=1 \\ r_{1}+1+\frac{m(m+1)}{2} \frac{m^{k-1}-1}{m-1} & \text { if } k>1,\end{cases}
$$

which proves the lemma for $i=1$. Specially we see that the lemma holds for $k=1$. Assume the lemma for all $k, 1 \leqq k \leqq K-1$. (2.6) and (2.7) gives

$$
\sum_{t=0}^{m^{i}-1} a_{t, K-1}(i)= \begin{cases}\frac{m(m+1)}{2} \frac{m^{K-1}-1}{m-1} & \text { if } i=1 \\ m \sum_{l=0}^{m^{i-1}-1} a_{m l, K-1}(i) & \text { if } i>1 .\end{cases}
$$

Hence from $(2.4) ; 1 \leqq i \leqq K$;

$$
\sum_{t=0}^{m^{i}-1} a_{t, K-1}(i) \equiv 0 \begin{cases}\left(\bmod m^{i}\right) & \text { if } m \text { is odd } \\ \left(\bmod m^{i} / 2\right) & \text { if } m \text { is even } .\end{cases}
$$


Let $2 \leqq i \leqq K$. If $\left[t / m^{i-2}\right]=m-1$ then $r_{i-1}(t)=m-1$, hence by the induction hypothesis

$$
a_{t, K-1}(i-1) \equiv 0 \quad\left(\bmod 2^{\mu(K-1)-1} m^{i-1}\right) .
$$

Now, $r_{i}=m-1$. Thus we have from (2.4) and (2.6) when $i>2$

$$
\begin{aligned}
\sum_{t=0}^{r_{2}+\ldots+r_{i} m^{i-2}-1} a_{t, K-1}(i-1) & =m \sum_{l=0}^{m^{i-3}(m-1)-1} a_{m l, K-1}(i-1)+\sum_{t=m^{i-2}(m-1)}^{r_{2}+\ldots+r_{i} m^{i-2}-1} a_{t, K-1}(i-1) \\
& \equiv 0 \begin{cases}\left(\bmod m^{i-1}\right) & \text { if } m \text { is odd } \\
\left(\bmod m^{i-1} / 2\right) & \text { if } m \text { is even } .\end{cases}
\end{aligned}
$$

If $i=2$

$$
\begin{aligned}
\sum_{t=0}^{r_{2}-1} a_{t, K-1}(1) & =\sum_{t=0}^{m-1} a_{t, K-1}(1)-a_{m-1, K-1}(1) \\
& \equiv 0 \begin{cases}(\bmod m) & \text { if } m \text { is odd } \\
(\bmod m / 2) & \text { if } m \text { is even }\end{cases}
\end{aligned}
$$

Hence (2.5) gives when $r_{i}=m-1$ and $2 \leqq i \leqq K$;

$$
a_{n, K}(i) \equiv 0 \begin{cases}\left(\bmod m^{i}\right) & \text { if } m \text { is odd } \\ \left(\bmod m^{i} / 2\right) & \text { if } m \text { is even }\end{cases}
$$

which completes the proof of the lemma.

From (2.1) and (2.2) we obtain

Hence

$$
s_{m, q}(m n) \equiv\left(r_{1}+1\right) s_{m, q-1}\left(m n_{1}\right) \quad(\bmod m) .
$$

$$
s_{m, q}(m n) \equiv \prod_{i=0}^{q-1}\left(\left[n / m^{i}\right]+1\right) \quad(\bmod m) .
$$

which proves Theorem 1 for $k=1$. Assume by induction Theorem 1 for all $k, 1 \leqq k \leqq K-1$.

We note that $\left[n / m^{j}\right] \equiv r_{j+1}(\bmod m)$. Put $1 \leqq i \leqq K ; K \leqq q$.

Suppose that $\lambda_{i}$ of the numbers $r_{l} ; l=1, \ldots, i$; are equal to $m-1$, hence $K-\lambda_{i} \leqq q-i$ where $K-\lambda_{i}$ denotes the number of $r_{l} ; l=i+1, \ldots, q$; equal to $m-1$. If $q-i>0$ we thus have from the induction hypothesis since $r_{j}\left(n_{i}\right)=r_{j+i}(n)$;

$$
s_{m, q-i}\left(m n_{i}\right) \equiv 0 \begin{cases}\left(\bmod m^{K-\lambda_{i}} / c_{K-\lambda_{i}}\right) & \text { if } \lambda_{i}>0 \\ \left(\bmod m^{K-1} / c_{K-1}\right) & \text { if } \lambda_{i}=0\end{cases}
$$

Note that $r_{i}=m-1$ if $i=\lambda_{i}$ or $i=K=q$. Let $1<i \leqq K$. By means of (2.4), (2.8) and the lemma we obtain

$$
a_{n, K}(i) s_{m, q-i}\left(m n_{i}\right) \equiv 0 \quad\left(\bmod m^{K} / c_{K}\right) .
$$


Thus from (2.3)

$$
s_{m, q}(m n) \equiv a_{n, K}(1) s_{m, q-1}\left(m n_{1}\right) \quad\left(\bmod m^{K} / c_{K}\right) .
$$

Hence if $\sigma$ denotes the smallest integer such that $r_{\sigma}=m-1 ; 1 \leqq \sigma<q$; we easily deduce

$$
s_{m, q}(m n) \equiv \prod_{i=0}^{\sigma-1} a_{n_{i}, K}(1) s_{m, q-\sigma}\left(m n_{\sigma}\right) \quad\left(\bmod m^{K} / c_{K}\right) .
$$

Now, since $r_{1}\left(n_{\sigma-1}\right)=r_{\sigma}(n)=m-1$ the lemma gives

$$
a_{n_{\sigma-1}, K}(1) \equiv 0 \begin{cases}(\bmod m) & \text { if } m \text { is odd } \\ (\bmod m / 2) & \text { if } m \text { is even },\end{cases}
$$

and from the induction hypothesis

$$
s_{m, q-\sigma}\left(m n_{\sigma}\right) \equiv 0 \quad\left(\bmod m^{K-1} / c_{K-1}\right) .
$$

This completes the proof of Theorem 1 .

If $k \leqq q$ Theorem 2 follows immediately from (1.1) when $i=1$. It remains to prove Theorem 2 in the case $k>q$.

From (2.2) we have

$$
s_{m, q}(m n-m)=\sum_{j=1}^{n} s_{m, q-1}(n-j) .
$$

By induction on $k$, it is now easily proved that there exist integers $b_{k}(j) ; 0 \leqq k \leqq q-1$; depending on $m$, such that

$$
s_{m, q}\left(m^{k+1} n-m\right)=\sum_{j=1}^{n} b_{k}(j) s_{m, q-(k+1)}(n-j),
$$

where

$$
b_{k}(j)= \begin{cases}1 & \text { if } k=0 \\ \sum_{i=1}^{m j} b_{k-1}(i) & \text { if } k>0 .\end{cases}
$$

There exist integers $\varrho_{k}(l) ; 1 \leqq k \leqq q-1$; depending on $m$, such that

$$
\sum_{l=1}^{k} \varrho_{k}(l) b_{k+1-l}(j)=\left(\begin{array}{l}
j \\
k
\end{array}\right) m^{\left(\begin{array}{c}
k+1 \\
2
\end{array}\right)},
$$

where $\varrho_{k}(1)=1$.

From (2.10) we see that (2.11) holds for $k=1$. Assume by induction that (2.11) holds for all $k, 1 \leqq k<K ; K \leqq q-1$; then

$$
\sum_{i=1}^{m j} \sum_{l=1}^{K-1} \varrho_{K-1}(l) b_{K-l}(i)=\left(\begin{array}{c}
m j+1 \\
K
\end{array}\right) m^{\left(\begin{array}{c}
K \\
2
\end{array}\right)},
$$

and from (2.10)

$$
\sum_{l=1}^{K-1} \varrho_{K-1}(l) b_{K+1-l}(j)=\left(\begin{array}{c}
j \\
K
\end{array}\right) m^{\left(\begin{array}{c}
K+1 \\
2
\end{array}\right)}+m^{\left(\begin{array}{c}
K \\
2
\end{array}\right)} \sum_{l=1}^{K-1}\left(\begin{array}{c}
j \\
K-l
\end{array}\right) v_{K, l},
$$


when observing that

$$
\left(\begin{array}{c}
m j+1 \\
K
\end{array}\right)
$$

being a polynomial in $j$ of degree $K \geqq 2$, can be written

$$
\left(\begin{array}{c}
m j+1 \\
K
\end{array}\right)=\left(\begin{array}{c}
j \\
K
\end{array}\right) m^{K}+\sum_{l=1}^{K-1}\left(\begin{array}{c}
j \\
K-l
\end{array}\right) v_{K, l},
$$

where $v_{K, l}$ are integers depending on $m$. Since

$$
\begin{aligned}
& \sum_{l=1}^{K-1} \sum_{t=1}^{l} m^{\left(\begin{array}{l}
K \\
2
\end{array}\right)-\left(\begin{array}{c}
K-t+1 \\
2
\end{array}\right)} v_{K, t} \varrho_{K-l}(l-t+1) b_{K-l}(j) \\
&=\sum_{l=1}^{K-1}\left(\begin{array}{c}
j \\
K-l
\end{array}\right) v_{K, l} m^{\left(\begin{array}{l}
K \\
2
\end{array}\right),}
\end{aligned}
$$

it is immediately seen that (2.11) holds for $k=K$ and that

$$
\varrho_{K}(l+1)=\varrho_{K-1}(l+1)-\sum_{t=1}^{l} m^{\left(\begin{array}{l}
K \\
2
\end{array}\right)-\left(\begin{array}{c}
K-t+1 \\
2
\end{array}\right)} v_{K, t} \varrho_{K-t}(l-t+1),
$$

for $1 \leqq l \leqq K-1$.

We put $\varrho_{K}(l+1)=0$ if $K<l+1$. Computing coefficients of $j^{K-1}$ on the two sides of (2.12)

$$
2 v_{K, 1}=(K-1) m^{K}-(K-3) m^{K-1} .
$$

From this and (2.13) we obtain

Hence

$$
\varrho_{K}(l+1) \equiv \varrho_{K-1}(l+1) \begin{cases}\left(\bmod m^{K-1}\right) & \text { if } m \text { is odd } \\ \left(\bmod m^{K-1} / 2\right) & \text { if } m \text { is even } .\end{cases}
$$

$$
\varrho_{K}(l+1) \equiv 0 \begin{cases}\left(\bmod m^{l}\right) & \text { if } m \text { is odd } \\ \left(\bmod m^{l} / 2\right) & \text { if } m \text { is even }\end{cases}
$$

Now we can prove that

$$
\sum_{j=1}^{m^{t} n} b_{k}(j) \equiv 0 \begin{cases}\left(\bmod m^{k+t}\right) & \text { if } m \text { is odd } \\ \left(\bmod m^{k+t} / 2^{k}\right) & \text { if } m \text { is even },\end{cases}
$$

when $0 \leqq k \leqq q-1$.

This is immediately satisfied for $k=0,1$. Assume by induction that (2.15) holds for all $k, 1 \leqq k<K ; K \leqq q-1$.

If $p$ is a prime we define the natural number $\psi$ by

$$
p^{\varphi} \mid(K+1) ! ; \quad p^{\varphi+1} \nmid(K+1) ! .
$$

Math. Scand. $37-6$ 


\section{Hence}

Thus

$$
\psi=\sum_{i=1}^{K-1}\left[K+1 / p^{i}\right] \leqq \sum_{i=1}^{K-1} i=\left(\begin{array}{c}
K \\
2
\end{array}\right)
$$

From (2.11) we have

$$
\begin{aligned}
\sum_{j=1}^{m^{t} n} b_{K}(j)+\sum_{l=2}^{K} \varrho_{K}(l) \sum_{j=1}^{m^{t_{n}}} b_{K+1-l}(j) & =\sum_{j=1}^{m^{t_{n}}}\left(\begin{array}{c}
j \\
K
\end{array}\right) m^{\left(\begin{array}{c}
K+1 \\
2
\end{array}\right)} \\
& =\left(\begin{array}{c}
m^{t} n+1 \\
K+1
\end{array}\right) m^{\left(\begin{array}{c}
K+1 \\
2
\end{array}\right)} .
\end{aligned}
$$

Hence from (2.14), (2.16) and the induction hypothesis

$$
\sum_{j=1}^{m^{t} n} b_{K}(j) \equiv 0 \begin{cases}\left(\bmod m^{K+t}\right) & \text { if } m \text { is odd } \\ \left(\bmod m^{K+t} / 2^{K}\right) & \text { if } m \text { is even }\end{cases}
$$

which completes the proof of (2.15).

Now, let $k>q$. From (2.9) and (2.15) we obtain

$$
\begin{aligned}
s_{m, q}\left(m^{k+1} n-m\right) & =s_{m, q}\left(m^{(q-1)+1} m^{k-(q-1)} n-m\right) \\
& =\sum_{l=1}^{m^{k-(q-1)} n} b_{q-1}(l) \equiv 0 \begin{cases}\left(\bmod m^{k}\right) & \text { if } m \text { is odd } \\
\left(\bmod m^{k} / 2^{q-1}\right) & \text { if } m \text { is even },\end{cases}
\end{aligned}
$$

which completes the proof of Theorem 2.

\section{REFERENCES}

1. G. E. Andrews, Congruence properties of the m-ary partition function, J. Number Theory 3 (1971), 104-110.

2. R. F. Churchhouse, Congruence properties of the binary partition function, Proc. Cambridge Philos. Soc. 66 (1969), 371-376.

3. G. Dirdal, On restricted m-ary partitions, Math. Scand. 37 (1975), 51-60.

4. H. Gupta, On m-ary partitions, Proc. Cambridge Philos. Soc. 71 (1972), 343-345.

5. O. Rödseth, Some arithmetical properties of m-ary partitions, Proc. Cambridge Philos. Soc. 68 (1970), 447-453. 